\title{
Recent Progress in CERN RD39: Radiation Hard Cryogenic Silicon Detectors for Applications in LHC Experiments and Their Future Upgrades
}

\author{
E. Tuominen, P. Anbinderis, T. Anbinderis, R. Bates, W. de Boer, E. Borchi, M. Bruzzi, C. Buttar, W. Chen,
} V. Cindro, S. Czellar, A. Dierlamm, V. Eremin, E. Gaubas, J. Härkönen, E. Heijne, I. Ilyashenko, V. Kalesinskas, M. J. Kortelainen, T. Lampén, Z. Li, P. Luukka, I. Mandic, D. Menichelli, M. Mikuz, O. Militaru, S. Mueller, T. Mäenpää, T. O. Niinikoski, V. O’Shea, C. Parkes, K. Piotrzkowski, S. Pirollo, P. Pusa, J. Räisänen, E. Tuovinen, J. Vaitkus, E. Verbitskaya, S. Väyrynen, and M. Zavrtanik

\begin{abstract}
CERN RD39 Collaboration develops radiation-hard cryogenic silicon detectors. Recently, we have demonstrated improved radiation hardness in novel Current Injected Detectors (CID). For detector characterization, we have applied cryogenic Transient Current Technique (C-TCT). In beam tests, heavily irradiated CID detector showed capability for particle detection. Our results show that the CID detectors are operational at the temperature $-50^{\circ} \mathrm{C}$ after the fluence of $1 \times 10^{16} 1 \mathrm{MeV}$ neutron equivalent $/ \mathrm{cm}^{2}$.
\end{abstract}

Index Terms-Cryogenic, current injected detector, radiation hard, silicon detector, transient current technique.

\section{INTRODUCTION}

C ERN RD39 Collaboration develops radiation-hard cryogenic silicon detectors for the applications of the CERN LHC experiments and their future upgrades. Radiation hardness

Manuscript received September 08, 2008; revised January 09, 2009. Current version published August 12, 2009. This work was supported in part by the Finnish Academy of Sciences.

E. Tuominen is with the Helsinki Institute of Physics, FI-00014 University of Helsinki, Finland (e-mail: eija.tuominen@helsinki.fi).

P. Anbinderis, T. Anbinderis, E. Gaubas, V. Kalesinskas, and J. Vaitkus are with the University of Vilnius, Institute of Materials Science and Applied Research, 2040 Vilnius, Lithuania.

R. Bates, C. Buttar, V. O'Shea, and C. Parkes are with the Department of Physics and Astronomy, University of Glasgow, Glasgow G12 8QQ, U.K..

W. de Boer, A. Dierlamm, and S. Mueller are with the IEKP Forschungszentrum Karlsruhe, 76344 Eggenstein-Leopoldshafen, Germany.

E. Borchi, M. Bruzzi, D. Menichelli, and S. Pirollo are with the Dipartimento di Energetica, Universitá di Firenze, I-50139 Firenze, Italy.

W. Chen and Z. Li are with the Brookhaven National Laboratory, Upton, NY 11973-5000 USA.

V. Cindro, I. Mandic, M. Mikuz, and M. Zavrtanik are with the Jozef Stefan Institute, Experimental Particle Physics Department, Ljubljana, Slovenia.

S. Czellar, J. Härkönen, M. J. Kortelainen, T. Lampén, P. Luukka, T. Mäenpää, and E. Tuovinen are with the Helsinki Institute of Physics, FI-00014 University of Helsinki, Finland.

V. Eremin, I. Ilyashenko, and E. Verbitskaya are with the Ioffe Physico-Technical Institute, Russian Academy of Sciences, St. Petersburg, Russia.

E. Heijne and T. O. Niinikoski are with CERN, CH-1211, Geneva, Switzerland.

O. Militaru and K. Piotrzkowski are with the Université Catholique de Louvain, FYNU, Louvain-la-Neuve, Belgium.

P. Pusa is with the Department of Physics, University of Liverpool, Liverpool L697ZE, U.K.

J. Räisänen and S. Väyrynen are with the Department of Physics, Division of Materials Physics, University of Helsinki, FI-00014 University of Helsinki, Finland.

Digital Object Identifier 10.1109/TNS.2009.2013950 up to the fluence of $1 \times 10^{16} 1 \mathrm{MeV}$ neutron equivalent $/ \mathrm{cm}^{2}$ is required in the future High Energy Physics (HEP) experiments, such as the upgrade of LHC, or Super-LHC, foreseen to be launched after some 10 years of LHC operation. Such fluence is beyond the radiation tolerance of currently existing silicon sensor technology.

The most important indication of the radiation hardness of a silicon particle detector is Charge Collection Efficiency (CCE). The total charge collected by a detector, $Q$, can be considered to be a product of three terms [1]:

$$
Q=Q_{0} \times \mathrm{CCE}_{\mathrm{GF}} \times \mathrm{CCE}_{t}
$$

$Q_{0}$ is the charge induced by the hitting particle or photon. The term $\mathrm{CCE}_{\mathrm{GF}}$ is a geometrical factor related to the detector depletion thickness, the weighting function (detector segmentation scheme) [2], [3] and the detector configuration (e.g., planar detectors or $3 \mathrm{~d}$ detector) [4]. The term $\mathrm{CCE}_{t}$ is a trapping factor. The trapping time constant is nearly independent on temperature. However, it depends strongly on the radiation fluence. At a fluence of $1 \times 10^{16} \mathrm{n} / \mathrm{cm}^{2}$, the trapping time can be as short as $0.2 \mathrm{~ns}$ [5].

As demonstrated by previous RD39 results [6], [7] $\mathrm{CCE}_{\mathrm{GF}}$ can be increased close to 1 by manipulating the electric field in the detector via current and/or charge injection at temperatures from $130 \mathrm{~K}$ to $150 \mathrm{~K}$. Since for the fluence less than $1 \times 10^{15} \mathrm{n} / \mathrm{cm}^{2}$, the trapping term $\mathrm{CCE}_{t}$ is less significant, $\mathrm{CCE}$ can be significantly improved by improving only $\mathrm{CCE}_{\mathrm{GF}}$ at $130 \mathrm{~K}-150 \mathrm{~K}$. However, for the extremely high fluence $(1 \times$ $10^{16} \mathrm{n} / \mathrm{cm}^{2}$ ) present in the environment of the LHC upgrade, the $\mathrm{CCE}_{t}$ is significant and affects greatly the CCE.

Radiation fluence $1 \times 10^{16} \mathrm{n} / \mathrm{cm}^{2}$ will deteriorate silicon detectors in two severe ways. First, the full depletion voltage $\left(V_{f d}\right)$ will become thousands of volts for a $300 \mu \mathrm{m}$ thick silicon detector operated at room temperature. Secondly, the charge carrier trapping will limit the charge collection depth to an effective range of $20 \mu \mathrm{m}$ to $30 \mu \mathrm{m}$ regardless of the detector depletion depth. In order to maintain an acceptable CCE in such a radiation environment, both these problems must be solved simultaneously.

The approach of CERN RD39 Collaboration to improve the detector radiation performance at very high fluencies is to re- 
alize controlled filling of trapping centers at low temperatures. The key of our approach is to use current injection inside the detector bulk to maintain the detector fully depleted after any fluencies, and to fill the traps to increase the trapping time constant that affects the CCE.

Recently, the two major activities within the CERN RD39 Collaboration have been the development of the radiation-hard Current Injected Detectors (CID) and the development of the Cryogenic Transient Current Technique (C-TCT) set-up and its application to detector characterization.

\section{CRyogenic Transient CurRent TeChniQue (C-TCT)}

We have constructed Transient Current Technique (TCT) and Charge Collection Efficiency (CCE) set-ups at CERN with temperature range from $35 \mathrm{~K}$ to $300 \mathrm{~K}$ (Cryogenic-TCT or $\mathrm{C}$-TCT). The interest in the extension of the temperature range below liquid nitrogen is important both in the development of Super-LHC tracking detectors and in the basic understanding of heavily radiation-damaged silicon.

The TCT measurement, originally presented in [8], is based on the detection of the dominant type of charge carriers, electrons or holes, which drift across the whole detector thickness after being excited by a photon. This is achieved by illuminating the front or the back implant of the detector with laser having a short absorption depth in silicon. The RD39 setup can be operated both with red laser $(670 \mathrm{~nm})$ that generates a cloud of electron-hole pairs very close to the front surface of the device, and with infrared laser $(1060 \mathrm{~nm})$ that generates electron-hole pairs throughout the entire thickness of the detector thus simulating the effect of a MIP (minimum ionizing particle).

When the front-side of a $\mathrm{p}^{+} / \mathrm{n}^{-} / \mathrm{n}^{+}$detector is illuminated with red laser, the holes move only few micrometers (resulting in a small signal) and are gathered to the $\mathrm{p}^{+}$-electrode so fast that the signal is damped by the rise-time of the data acquisition electronic. Therefore, the measured signal is mainly coming from the electrons that travel a longer distance through the silicon bulk. Correspondingly, when the front surface of a $\mathrm{n}^{+} / \mathrm{p}^{-} / \mathrm{p}^{+}$detector is illuminated, mainly the current from holes is measured. In the case of infrared laser, the measured current consists of both electrons and holes.

The measured signal, i.e., the TCT-transient, can be expressed as:

$$
i(t) \propto \frac{Q_{0}}{d} \times \mu \times E(x) \times e^{-\frac{t}{\tau_{\text {trapping }}}} .
$$

Here $Q_{0}$ is the induced charge, $d$ is the thickness of the detector, $\mu_{n, p}$ is the mobility of the charge carriers, $E(x)$ is the electric field as a function of distance, $t$ is the time, and $\tau_{\text {trapping }}$ is the trapping time constant. By analyzing the transient generated by red laser, it is possible to extract the full depletion voltage [9], effective trapping time [10], [11], electric field distribution [12], [13] and the sign of the space charge in the silicon bulk [6], [8].

As an example, in the specific case of Magnetic Czochralski $\mathrm{n}$ - and p-type silicon (MCz-Si) detectors irradiated with $24 \mathrm{GeV}$ protons, so called double-peak TCT-signal has been observed

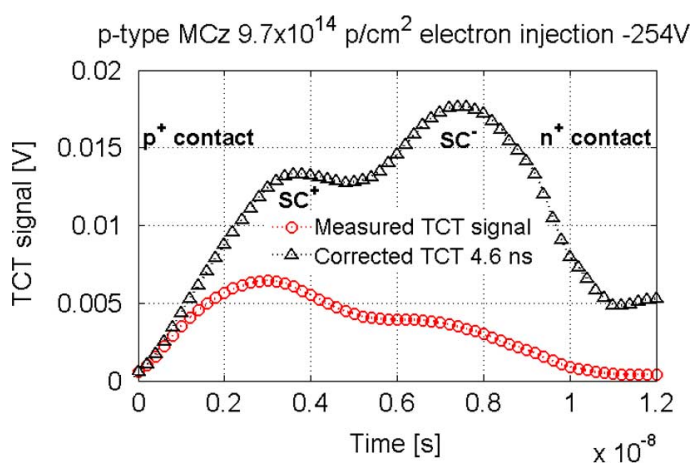

Fig. 1. Douple-peak TCT signal measured from p-type Czochralski silicon detector $\left(\mathrm{n}^{+} / \mathrm{p}^{-} / \mathrm{p}^{+}\right)$irradiated $9.7 \times 10^{14} \mathrm{p} / \mathrm{cm}^{2}$ with $24 \mathrm{GeV}$ protons. The measurement was done by red laser and back side illumination.

[13], see Fig. 1. The problem in analyzing the electric field distribution $E(x)$ in MCz-Si detectors is that Space Charge Sign Inversion (SCSI) takes place at such a high fluence $(>1 \times$ $10^{14} \mathrm{n}_{\mathrm{eq}} / \mathrm{cm}^{2}$ ) that the trapping effects must be taken into account. Thus, more systematic TCT studies, including electron and hole injections into both n- and p-type sensors, are needed to explain the electric field distribution in heavily irradiated $\mathrm{MCz}-\mathrm{Si}$ sensors.

Unlike in Float Zone silicon (Fz-Si), the two peaks observed in $\mathrm{n}$ and p-type $\mathrm{MCz}-\mathrm{Si}$ are almost similar (after trapping corrections), indicating half positive space charge (+SC) and half negative space charge (-SC) in the detector, especially at high fluencies [14].

\section{CURRENT INJECTED DETECTORS (CID)}

We have developed advanced radiation-hard detectors using charge or current injection. Two approaches have been applied: the electric field manipulation and self-limited current injection effect realized in CID Detectors [15], [16]. The method of electric field manipulation is based on the reduction of the negative effective doping concentration (Neff) by injecting holes from the $\mathrm{n}^{+}$contact of a $\mathrm{p}^{+} / \mathrm{n}^{-} / \mathrm{n}^{+}$detector.

The effective doping concentration is the difference between ionized acceptor $(\mathrm{Na})$ and donor $(\mathrm{Nd})$ concentrations. At low temperatures, injected holes are captured by radiation induced defects. This results in the accumulation of positive space charge and corresponding reduction in the absolute value of $N e f f$ from its initial negative value of $-N e f f O$. At a certain level of injected current, the electric field becomes almost uniform meaning that the INeffI can be reduced close to zero. When a standard $\mathrm{p}^{+} / \mathrm{n}^{-} / \mathrm{n}^{+}$detector is reverse-biased, the advantage of the injection is related to the electric field distribution. It has been shown that the optimal injected current depends on the operational voltage and the fluence of the detector [15]. Therefore, the current needs to be adjusted at any changes of operational parameters.

In a CID, the electric field inside the detector is controlled by injected current, which is limited by the space charge. This leads to a continuous electric field through the detector at any operating voltage regardless of the radiation fluence.

To compensate the negative $N_{\text {effo }}$ and to overcome the collapse of electric field under irradiation, self-limited injection of 


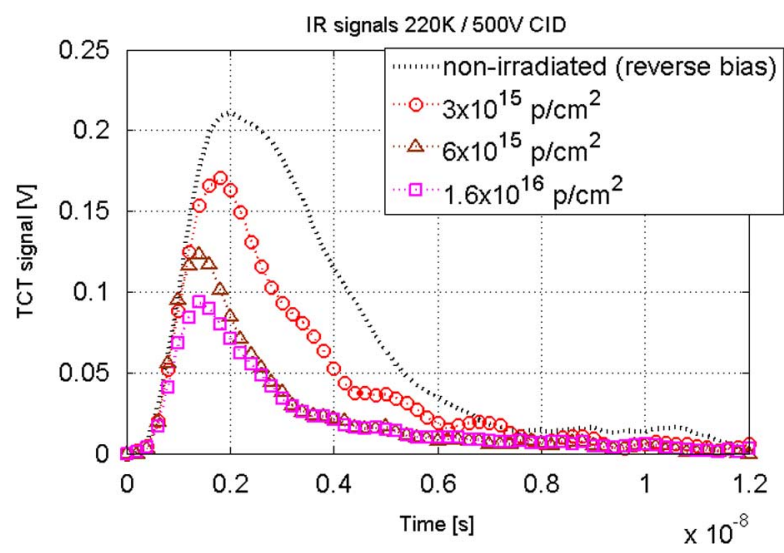

Fig. 2. TCT-signals from three irradiated silicon samples compared with a nonirradiated sample.

holes is applied through the $\mathrm{n}+$ contact. The trapping centers produced e.g., by particle radiation are uniformly distributed in the detector bulk. When a bias voltage is applied, the bulk is fully or partially depleted depending on the electric field distribution defined by the density of charged donors and acceptors. To compensate the negative $N_{\text {effo }}$ and to overcome the collapse of electric field under irradiation, the injection of the hole current is applied through the $\mathrm{n}^{+}$contact. The injected holes are trapped by deep levels resulting in reduced negative effective concentration and more uniform electric field distribution. The Poisson equation, Ohm's law and rate equation define the steady state electric field distribution in semiconductor bulk. A detailed mathematical description of the physics in CID detectors can be found in [15].

The charge collected in a $300 \mu \mathrm{m}$ thick CID at $1 \times 10^{16} \mathrm{n} / \mathrm{cm}^{2}$ is estimated to be equivalent to 5000 electrons at $500 \mathrm{~V}$ bias, as shown by our results. This charge is more than in a standard detector hypothetically fully depleted with thousands of volts. An important advantage of CID is that at some given operating voltage, the current decreases with increasing irradiation fluence. In a segmented detector this would result in decreasing shot noise with respect of the increasing fluence. Additionally, with the CID concept it is possible to increase the absolute value of the collected charge when the concentration of active trapping centers is reduced by cooling to low temperatures.

\section{Characterizing CID BY C-TCT}

Our results show that the effective space charge in silicon detectors can be manipulated by charge injection and temperature. Fig. 2 presents TCT measurements made of irradiated silicon pad detectors operated at CID mode. Three samples were irradiated by $24 \mathrm{GeV} / \mathrm{c}$ protons up to the fluence of $1.6 \times 10^{16} \mathrm{p} / \mathrm{cm}^{2}$ [17]. All the measurements were done at $220 \mathrm{~K}$. The uppermost solid-line curve is a non-irradiated reference detector made on MCz-Si material. The CID p $\mathrm{p}^{+} / \mathrm{n}^{-} / \mathrm{n}^{+}$detector structures were also made of $\mathrm{MCz}-\mathrm{Si}$. The bias of the CID detectors during the measurement was $500 \mathrm{~V}$. At fluencies $>10^{15} \mathrm{n}_{\mathrm{eq}} / \mathrm{cm}^{2}$, the depletion voltage $\left(V_{f d}\right)$ would be more than $1000 \mathrm{~V}$ at temperatures from $0^{\circ}$ to $-30^{\circ} \mathrm{C}$ [15], [18].

As shown in Fig. 2, if $100 \%$ CCE is assumed for the nonirradiated reference detector, the CID detector that has received

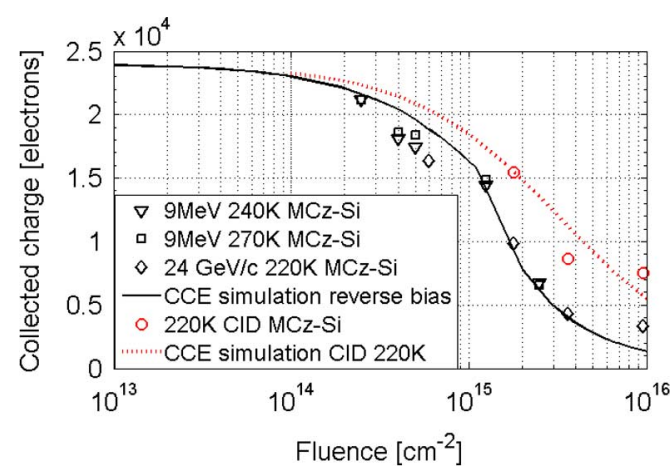

Fig. 3. Collected charge vs fluence of $9 \mathrm{MeV}$ and $24 \mathrm{GeV} / \mathrm{c}$ proton irradiated $\mathrm{MCz}-\mathrm{Si}$ detectors compared to simulations.

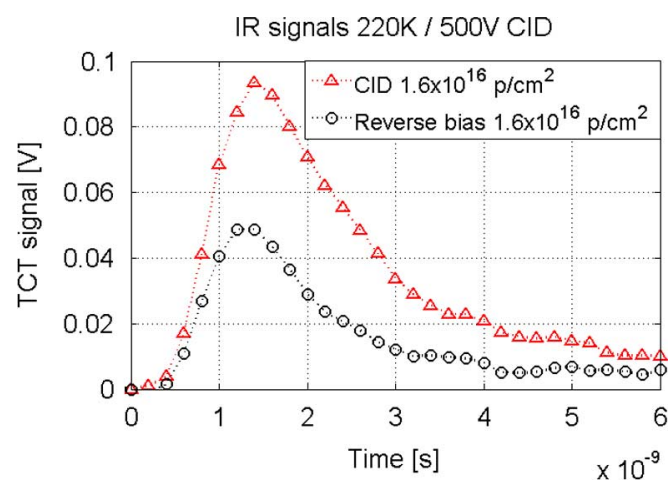

Fig. 4. Infrared laser signals of $1.6 \times 10^{16} \mathrm{p} / \mathrm{cm}^{2} 24 \mathrm{GeV} / \mathrm{c}$ proton irradiated $\mathrm{MCz}-\mathrm{Si}$ detector in CID mode $(\Delta)$ and under reverse bias $(0)$. Both measurements were done at $220 \mathrm{~K}$ and without evacuating the vacuum of the sample holder chamber of the C-TCT system and without changing the laser settings.

a fluence of $3 \times 10^{15} \mathrm{p} / \mathrm{cm}^{2}$ would have about $45 \%$ CCE. Even the heavily irradiated detector that has received a dose of $1.6 \times$ $10^{16} \mathrm{p} / \mathrm{cm}^{2}$ shows about $27 \% \mathrm{CCE}$.

Fig. 3 shows collected charge as a function of irradiation fluence for low and high energy proton irradiated $300 \mu \mathrm{m}$ thick $\mathrm{MCz}-\mathrm{Si}$ detectors. The data points below $1 \times 10^{15} \mathrm{n} / \mathrm{cm}^{2}$ were measured at $240 \mathrm{~K}$ and $270 \mathrm{~K}$ and the data points above $1 \times$ $10^{15} \mathrm{n} / \mathrm{cm}^{2}$ at $220 \mathrm{~K}$. The dashed lines are CCE simulations of (1). The simulation of CCE assumes linear trapping, i.e., at the fluencies $1 \times 10^{14} \mathrm{n}_{\mathrm{eq}} / \mathrm{cm}^{2}, 1 \times 10^{15} \mathrm{n}_{\mathrm{eq}} / \mathrm{cm}^{2}$ and $1 \times$ $10^{16} \mathrm{n}_{\mathrm{eq}} / \mathrm{cm}^{2}$ the values for $\tau_{t}$ are $10 \mathrm{~ns}, 1 \mathrm{~ns}$ and $0.1 \mathrm{~ns}$, respectively [19], [11]. The threshold of an acceptable signal-to-noise for typical readout in terms of electron equivalent is about 7000 electrons. The simulations and measurements were done at reverse bias of $500 \mathrm{~V}$ and at CID operation of $500 \mathrm{~V}$ for standard MCz-Si and CID MCz-Si detectors, respectively. As seen in Fig. 3, the CCE starts to decrease rapidly at the fluence of about $7 \times 10^{14} \mathrm{n}_{\mathrm{eq}} / \mathrm{cm}^{2}$. This is the fluence where the $V_{f d}$ of a $300 \mu \mathrm{m}$ thick MCz-Si detector reaches $500 \mathrm{~V}$ and the $\mathrm{CCE}_{\mathrm{GF}}$ in (1) starts to decrease. The simulation of CID detector is based on the condition that $\mathrm{CCE}_{\mathrm{GF}}=1$, and calculating the $\mathrm{CCE}_{t}$ with square root dependent electric field distribution across the detector bulk.

The collected charge after heavy irradiation is about two times higher in the CID operation than in the reverse bias operation. This can qualitatively be seen in Fig. 4. 


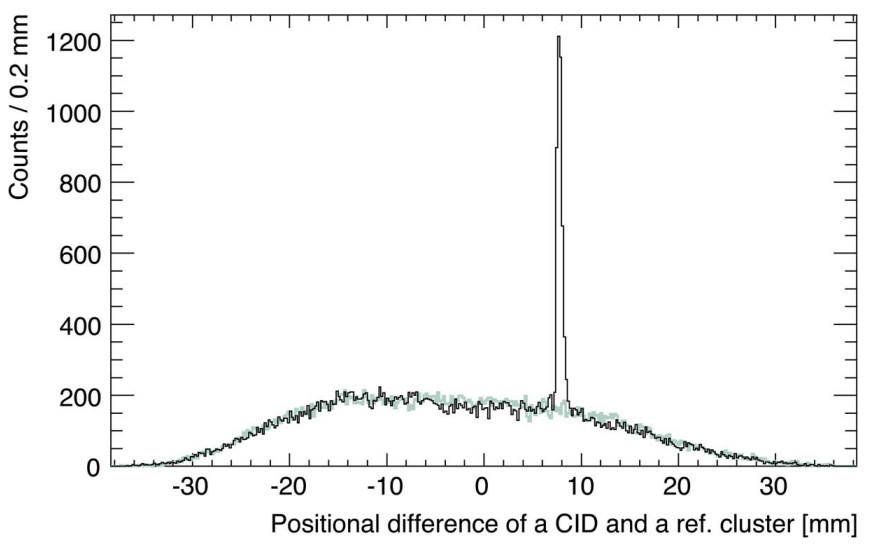

Fig. 5. Distribution of positional differences of cluster pairs of the heavily irradiated CID detector and a reference detector. The dark graph comprises all combinations of one CID cluster and one cluster from the reference detector in one event. In the light graph, clusters are taken from different events.

There is a good agreement between the measured and estimated CCE values of CID detectors. As shown by the experimental results in Figs. 3 and 4, the CID operation would provide about two times higher CCE at very high fluencies, namely above $2 \times 10^{15} \mathrm{n}_{\mathrm{eq}} / \mathrm{cm}^{2}$. As standard detectors, also CID detectors are influenced by the trapping. Therefore, the CCE of CIDs also decreases with respect to increasing irradiation fluence. The improved CCE is mainly due to the electric field, which extends through the entire bulk at any operating voltage and irradiation fluence. The higher CCE is achieved already at the temperatures of $-50^{\circ} \mathrm{C}$. It is worth noting that it is possible to operate normal AC-coupled strip detector structures as CID.

\section{Characterizing CID IN A TEST BEAM}

During summer 2008, the particle detection performance of an irradiated segmented CID detector was studied for the very first time. This was done at CERN H2 test beam station with $225 \mathrm{GeV}$ muons. The reference telescope was the Silicon Beam Telescope (SiBT) on the CERN H2 beam line, developed as a common effort between various institutes [20]. The SiBT consists of eight position sensitive silicon strip detectors, modified CMS data acquisition system and a cool box where the reference detectors can be cooled down to $-20^{\circ} \mathrm{C}$. For separately cooling the CID detectors down to their operating temperature $-50^{\circ} \mathrm{C}$, a special cooling plate was designed, constructed and placed immediately in front of the reference telescope in the muon beam. The details of the SiBT data analysis method are explained in [21].

The CID detector was irradiated with $26 \mathrm{MeV}$ low energy protons up to the dose of the $3 \times 10^{15} \mathrm{n} / \mathrm{cm}^{2}$. The first monitoring plots and the related preliminary data analysis suggest that the heavily irradiated CID detector is capable of detection of particle tracks. The black curve in Fig. 5 depicts the difference between the position of a cluster detected by the CID detector and a cluster detected by the reference detector, when all this kind of cluster pairs within the same event are considered. The parallel muon tracks generate clusters with same positional difference at the CID and the reference detector, which can be seen as the narrow peak of the figure. Failure to reconstruct cluster at either of these detectors and existence of noise clusters leads to the wide background distribution.

For comparison, a similar graph has been made with the difference that clusters for the reference detector are from event $N$, and clusters for the CID detector are from event $N+2$, see Fig. 5. This eliminates the correlation caused by the muon tracks. Hence, the narrow peak is clearly generated by the muon tracks.

Fig. 5 suggests that there are many more noise clusters in the background than real clusters in the narrow peak. However, cluster reconstruction has not been optimized for this graph, and further analysis is needed before conclusions are made. The width of the narrow peak is determined by the resolutions of the CID detector and the reference detector as well as by the angular spread of the muon tracks and the fact that the detectors have not been aligned. Therefore it cannot be used as an estimate for the resolution of the CID detector.

Although these preliminary results are very promising, a complete offline analysis with track reconstruction with all reference detectors is needed before drawing conclusions about the particle detection performance and radiation tolerance of the novel concept of CID.

\section{CONCLUSION}

RD39 Collaboration has developed advanced radiation-hard detectors using charge or current injection. Two approaches have been applied: the electric field manipulation and self-limited current injection effect realized in CID Detector. The charge collected in $300 \mu \mathrm{m}$ thick CID at $1 \times 10^{16} \mathrm{~cm}^{-2}$ is estimated to be equivalent of 5000 electrons at the $500 \mathrm{~V}$ bias. This is more than in a standard detector hypothetically fully depleted with thousands of volts. The elevated CCE is observed already at $220 \mathrm{~K}$. The current-voltage and CCE properties of CIDs have been confirmed by many independent measurements at RD39 member laboratories. We observed that standard $\mathrm{p}^{+} / \mathrm{n}^{-} / \mathrm{n}^{+}$and $\mathrm{n}^{+} / \mathrm{p}^{-} / \mathrm{p}^{+}$silicon detectors can be operated at current injection mode with dominating injection from one of the contacts.

In summer 2008, the particle detection performance of an irradiated CID detector was studied for the very first time. According to the first results, the CID detector shows great potential for particle detection after heavy irradiation.

The processing technology or silicon properties do not influence the Current Injected Detector performance. Thus, the CID is potentially a very cost effective solution for the challenging radiation environment of the future high luminosity High Energy Physics experiments.

\section{ACKNOWLEDGMENT}

The authors would like to thank the colleagues from the Silicon Beam Telescope Collaboration for providing the CID detector performance measurements in a particle beam.

\section{REFERENCES}

[1] E. Verbitskaya et al., "The effect of charge collection recovery in silicon p-n junction detectors irradiated by different particles," Nucl. Instr. and Meth. A., vol. 514, pp. 47-61, 2003. 
[2] E. Gatti, G. Padovini, and V. Radeka, "Signal evaluation in multielectrode radiation detectors by means of a time dependent weighting vector," Nucl. Instr. and Meth., vol. 193, pp. 651-653, 1982.

[3] V. Radeka, "Low-noise techniques in detectors," Ann. Rev. Nucl. Part. Sci., vol. 38, pp. 217-277, 1988.

[4] S. Parker, C. Kenney, and J. Segal, "3D-A proposed new architecture for solid-state radiation detectors," Nucl. Instr. and Meth. A., vol. 395, pp. 328-343, 1997.

[5] H. W. Kraner et al., "The use of the signal current pulse shape to study the internal electric field profile and trapping effects in neutron damaged silicon detector,' Nucl. Instr. and Meth. A., vol. 326, pp. 350-356, 1993.

[6] Z. Li, C. J. Li, V. Eremin, and E. Verbitskaya, "Direct observation and measurements of neutron-induced deep levels responsible for $\mathrm{N}_{\text {eff }}$ changes in high-resistivity silicon detectors using TCT," Nucl. Instr. And Meth. A., vol. 388, pp. 297-307, 1997.

[7] E. Verbitskaya et al., "Optimization of electric field distribution by free carrier injection in silicon detectors operated at low temperatures," IEEE Trans. Nucl. Sci., vol. 49, no. 1, pp. 258-263, 2002.

[8] V. Eremin, N. Strokan, E. Verbitskaya, and Z. Li, "Development of transient current and charge techniques for the measurement of effective net concentration of ionized charges (Neff) in the space charge region of p-n junction detectors," Nucl. Instr. and Meth. A., vol. 372, pp. 388-398, 1996.

[9] B. Dezillie, V. Eremin, Z. Li, and E. Verbitskaya, "Polarization of silicon detectors by minimum ionizing particles," Nucl. Instr. and Meth. A., vol. 452, pp. 440-453, 2000.

[10] T. J. Brodbeck, A. Chilingarov, T. Sloan, E. Fretwurst, M. Kuhnke, and G. Lindstroem, "A new method of carrier trapping time measurement," Nucl. Instr. and Meth. A., vol. 455, pp. 645-655, 2000.

[11] G. Kramberger, V. Cindro, I. Mandic, M. Mikuz, and M. Zavrtanik, "Determination of effective trapping times for electrons and holes in irradiated silicon," Nucl. Instr. and Meth. A., vol. 476, pp. 645-651, 2002.
[12] V. Eremin, Z. Li, S. Roe, G. Ruggiero, and E. Verbitskaya, "Double peak electric field distortion in heavily irradiated silicon strip detectors," Nucl. Instr. and Meth. A., vol. 535, pp. 622-631, 2004.

[13] E. Verbitskaya, V. Eremin, Z. Li, J. Harkonen, and M. Bruzzi, "Concept of double peak electric field distribution in the development of radiation hard silicon detectors," Nucl. Instr. and Meth. A., vol. 583, pp. 77-86, 2007.

[14] Z. Li et al., "Systematic TCT investigation of equal-double-junctions in $24 \mathrm{GeV}$ proton irradiated MCZ n and p-type Si detectors," in 12th RD50 Workshop, Ljubljana, Slovenia, June 2-4, 2008, presented in.

[15] V. Eremin et al., "Current injected detectors (CID)-a new approach for detector operation in very high radiation environment," in IEEE NSS Conference Record, 2004, vol. 3, pp. 2003-2006.

[16] V. Eremin, J. Härkönen, Z. Li, and E. Verbitskaya, "Current injected detectors at super-LHC program," Nucl. Instr. and Meth. A., vol. 583, pp. 91-98, 2007.

[17] E. Tuovinen et al., "Czochralski silicon detectors irradiated with 24 GeV/c and $10 \mathrm{MeV}$ protons," Nucl. Instr. and Meth. A., vol. 568, pp. 83-88, 2006.

[18] S. Väyrynen et al., "Setup for irradiation and characterization of materials and Si particle detectors at cryogenic temperatures," Nucl. Instr. and Meth. A., vol. 572, pp. 978-984, 2007.

[19] CERN RD50 Collaboration RD50 Status Report CERN-LHCC-2005037.

[20] T. Mäenpää et al., "Silicon beam telescope for LHC upgrade tests," Nucl. Instr. and Meth. A., vol. 593, pp. 523-529, 2008.

[21] M. J. Kortelainen, T. Lampén, H. Moilanen, and T. Mäenpää, “Off-line calibration and data analysis for the silicon beam telescope at the CERN H2 beam," Nucl. Instr. and Meth., submitted for publication. 